\title{
Cosmic ray dose monitoring using RadFET sensors of the Rosetta instruments SESAME and COSIMA
}

\author{
Peter Falke ${ }^{a}$, Hans-Herbert Fischer ${ }^{a}{ }^{*}$, Klaus J. Seidensticker ${ }^{b}$, Klaus Thiel ${ }^{c}$, \\ Henning Fischer ${ }^{\mathrm{d}}$, Martin Hilchenbach ${ }^{\mathrm{d}}$, Hartmut Henkel ${ }^{\mathrm{e}}$, Andreas Koch ${ }^{\mathrm{e}}$ \\ a DLR, Raumflugbetrieb und Astronautentraining, Microgravity User Support Center, Cologne, Germany \\ ${ }^{\mathrm{b}}$ DLR, Institut für Planetenforschung, Berlin, Germany \\ ${ }^{\mathrm{c}}$ Universität Köln, Abt. Nuklearchemie, Cologne, Germany \\ d Max-Planck-Institut für Sonnensystemforschung, Göttingen, Germany \\ e von Hoerner \& Sulger GmbH, Schwetzingen, Germany
}

\section{A R T I C L E I N F O}

\section{Article history:}

Received 19 October 2015

Received in revised form

4 February 2016

Accepted 1 March 2016

Available online 3 March 2016

\section{Keywords:}

RadFET

Ionising dose

Rosetta

Philae

COSIMA

SESAME

\begin{abstract}
A B S T R A C T
On its more than 10 years journey to comet 67P/Churyumov-Gerasimenko, Rosetta carried RadFET ionising dose monitors in the central electronics of the orbiter instrument COSIMA and the lander instrument SESAME. The readings of the dosimeters were corrected for the temperature of the devices during measurements. Because the sensitivity of RadFETs depends on the energy of impinging charged particles, a mean efficiency factor for the prevalent proton radiation was determined by applying nine efficiency models to proton energy spectra of Rosetta's radiation environment.

The resulting dose profiles show linear increases of the accumulated dose with time, mainly caused by galactic cosmic radiation, and the arrival of two solar particle events in 2005 . The accumulated dose (in Silicon) during 3909 days in space from 2004-03-02 to 2014-11-14 was 3.2 \pm 0.6 Gy in case of COSIMA and $1.9 \pm 0.4 \mathrm{~Gy}$ for SESAME. The deviation of the two measurements is mainly due to the solar particle event in September 2005, which had a $5.3 \pm 1.0$ times stronger impact on the COSIMA RadFET. Measured dose levels are one order of magnitude lower than those expected before launch for not being exceeded on the $90 \%$ confidence level, which is mainly due to the low solar activity during the mission so far.
\end{abstract}

(c) 2016 IAA. Published by Elsevier Ltd. All rights reserved.

\section{Introduction}

The Rosetta spacecraft was launched in March 2004 and it arrived at comet $67 \mathrm{P} /$ Churyumov-Gerasimenko (henceforth $67 \mathrm{P} / \mathrm{CG}$ ) in August 2014. The complex spacecraft trajectory extended to Jupiter's orbit and included three Earth flybys (in March 2005, November 2007, and November 2009) and a further gravity assist manoeuvre at Mars (February 2007). After a joint cruise of about 6.6 billion $\mathrm{km}$, the lander Philae was detached from the main spacecraft (henceforth orbiter) in November 2014. Philae executed scientific measurements on the cometary surface during its 3-days First Science Sequence. Orbiter observations of 67P/CG will continue until September 2016.

Several sources of energetic charged particles contribute to the radiation exposure of a spacecraft (see [1] for a general description of the radiation environment in space). Rosetta passed the radiation belts of the Earth during launch phase and the flybys.

\footnotetext{
* Corresponding author.

E-mail address: Hans-Herbert.Fischer@dlr.de (H.-H. Fischer).
}

Regarding ionising dose in central electronics, the short encounters with the trapped ions and electrons are less significant than solar cosmic rays (SCR) and the low, but continuous background of galactic cosmic rays (GCR).

Solar cosmic rays are composed of many smaller and a few large solar particle events (SPEs). During SPEs, the Sun emits charged particles (mainly protons), which become accelerated, are guided by the field lines of the interplanetary magnetic field and can eventually arrive at the spacecraft. The energies of SCR are generally in the range of a tenth to a few hundred MeV/nucleon. Solar particle events last few hours to several days and their magnitudes are extremely different. During a long-term mission a number of SPEs is expected, but one strong solar particle event can account for almost the complete ionising dose actually received. The frequency of large solar particle events is related to the solar cycle, but the occurrence of single SPEs cannot be predicted. Statistical models are used to calculate the maximum SCR fluences which will likely not be exceeded on a certain confidence level.

Solar activity also modulates the flux of high-energy charged particles which enter the solar system from outside [2]. These 
particles are effectively shielded by the solar wind and thus the fluxes of galactic cosmic rays and SCR are anti-correlated. GCR contains protons (nearly $90 \%$ of the atomic nuclei), alpha particles $(\approx 10 \%)$ and about $1 \%$ heavier ions [3] with broad energy spectra peaking at about $1 \mathrm{GeV}$ in case of protons (and at several $100 \mathrm{MeV} /$ nucleon in case of the heavier particles). The high-energy GCR have larger ranges in matter than SCR and, considering dose effects only, become generally important for the heavier shielded parts of a spacecraft. The relative importance of GCR and SCR can, however, be time-dependent for a long-term mission like Rosetta, considering the variability of solar activity and the changing distances to the Sun.

To avoid severe degradation or even the loss of a component, the expected dose levels are calculated for each mission and spacecraft components are selected according to the required radiation hardness. The analyses of Rosetta's radiation environment, first performed for the journey to comet 46P/Wirtanen and later updated for 67P/CG, were based on the available SCR and GCR models before launch. The results depend on the applied confidence levels and the way how the complex trajectories to the target comets were considered.

Radiation hard electronics are a relevant cost factor for space missions and precise knowledge of the expected dose is desirable before launch. The reliability of statistical models applied for the prediction can be assessed and the confidence in new models improved by comparing the predicted dose levels with those actually measured during missions. In this paper, we report on in situ measurements of the ionising dose in central electronics of Philae's payload SESAME [4] and of the orbiter instrument COSIMA [5]. The used RadFET dosimeters were provided by ESA, mounted by the electronics contractor of both instruments (von Hoerner \& Sulger $\mathrm{GmbH}$ ) and operated by the respective instrument teams. Like several other ESA spacecraft, Rosetta was equipped additionally with a Standard Radiation Environment Monitor (SREM), which is capable to record proton and electron fluxes in different energy channels $[6,7]$.

RadFETs are a special kind of field-effect transistors (FETs), developed to be particularly sensitive to ionising radiation. Their small size, the low power consumption and the simplicity of the readout circuit make them attractive for use in space missions. A major drawback is the dependency of the sensitivity on the energy of the prevalent proton radiation, which is not precisely known for each device and which requires additional information on the proton spectra. Furthermore, the temperature of the devices must be monitored to apply corrections to the dose signals.

\section{Properties of RadFET dosimeters}

The RadFETs used for COSIMA and SESAME are based on p-channel metal oxide semiconductor field-effect transistors (pMOSFET). P-type FETs consist of an n-doped Silicon substrate, where two p-doped terminals (source and drain) are inserted. The third terminal, the gate, is connected to the substrate by an insulating $\mathrm{SiO}_{2}$ layer and situated between source and drain. By applying a negative voltage to the gate, a conducting channel can be inducted into the substrate between source and drain, if the voltage exceeds the threshold voltage $U_{\mathrm{T}}$. RadFETs exhibit a thick insulating layer at the gate and are produced to have a high density of trapping centres in the insulator, where ionizing radiation produces electron-hole pairs. The holes are then trapped, whereby the threshold voltage gets more negative (see [8] and references therein).

The threshold voltage can be measured by applying a constant drain current and measuring the voltage between drain and source. After turning on the current, the voltage signal might drift within the first minute due to release or attachment of charges at the border between substrate and insulating layer. A long term fading is possible for RadFETs as well due to changes in the number of trapped charges over time. Fading can cause an apparent increase or decrease of the accumulated dose, depending on trapping properties of the RadFET and the time of the RadFET read-out after irradiation. A RadFET out of the same manufacturing cycle as the devices mounted on Rosetta has been examined in [8] with long-term measurements (up to 80 days after irradiations with protons and ${ }^{60} \mathrm{Co}$ gamma rays). Considering the environmental conditions of SESAME electronics during flight (low dose rate, low non-operational temperatures), an overestimation of several percent of the accumulated dose was expected. On the other hand, Haran et al. [9] used a RadFET from the same manufacturer and obtained a 5\% decrease of the dose signal after one month.

The response of the RadFET depends on the radiation type and its energy [10] mainly due to the density of electron-hole pairs created by the impinging particles or photons and different recombination processes.

In general it is not possible to set the drain current such that counteracting temperature effects in the devices are balanced. The optimum current value at the so-called ZTC (zero temperature coefficient) point might even change from readout to readout after annealing [9].

COSIMA and SESAME central electronics includes "400 nm implanted" devices [11] from NMRC (Cork, Ireland; now Tyndall National Institute) out of the same manufacturing cycle [8]. Both instruments used the "type 1 " transistor of the device, which has a width to length ratio of $300 / 50[8,12]$.

\section{Data readout and device temperatures}

Both instruments read out their RadFETs by applying a constant drain current of $-10 \mu \mathrm{A}$ and measuring the voltage between drain and source. COSIMA measurements were taken $20 \mathrm{~s}$ after turning on the supply voltage to reduce the drift. SESAME measured in six different readout variants with waiting periods between $1 \mathrm{~s}$ and above $1 \mathrm{~min}$. The measurement precision of the readout circuit in case of COSIMA is $1.2 \mathrm{mV}$, for SESAME $2 \mathrm{mV}$.

The device temperatures have been measured by a Pt1000 about $1.4 \mathrm{~cm}$ away from the RadFET on the same printed circuit board for SESAME and a temperature sensor glued onto the RadFET by COSIMA. During periods were no readouts took place, no voltage has been applied to the RadFETs and the temperatures were between $-35^{\circ} \mathrm{C}$ and $-24^{\circ} \mathrm{C}$ in case of SESAME. COSIMA non-operational temperatures were slightly above $0{ }^{\circ} \mathrm{C}$, except for the deep space hibernation period during which temperatures were expected to be down to $-20^{\circ} \mathrm{C}$ [13].

\section{Data analysis}

\subsection{Coverage and data processing}

The dataset covers ground test data and flight data, which were obtained in a time period from Rosetta's launch on 2004-03-02 up to 2015-05-26 in case of COSIMA and up to 2014-11-14, towards the end of Philae's First Science Sequence, for SESAME. The distance of Rosetta to the Sun was between 0.9 and $5.3 \mathrm{AU}$ during this period. Measurements took place roughly every six months. Between June 2011 and January 2014, Rosetta was in deep space hibernation and no measurements took place [14]. After arrival at the comet, the COSIMA measurements were executed almost permanently.

The data points have been grouped in sub-samples with 
durations below two weeks in order to reduce the statistical errors, assuming a constant dose signal for the two weeks.

\subsection{Temperature dependency of the signal}

The threshold voltage is expected to be linearly dependent on the temperature $T$ of the RadFET [8] according to $U_{\mathrm{T}}(T)=\kappa \cdot T\left[{ }^{\circ} \mathrm{C}\right]+U_{\mathrm{T}}\left(T=0{ }^{\circ} \mathrm{C}\right)$, where $\kappa$ is the temperature coefficient and $U_{\mathrm{T}}\left(T=0{ }^{\circ} \mathrm{C}\right)$ the threshold voltage at a temperature of $0{ }^{\circ} \mathrm{C}$ for a given point in time. The temperature coefficients have been determined by a linear regression to the sub-samples with the highest temperature range and a sufficient amount of data points for both instruments. For COSIMA one calibration was used and for SESAME two, which have been associated to the six readout variants. The obtained temperature coefficients are $0.404 \pm 0.002 \mathrm{mV} / \mathrm{K}$ for COSIMA and for the two SESAME calibrations $0.121 \pm 0.002 \mathrm{mV} / \mathrm{K}$ and $0.200 \pm 0.002 \mathrm{mV} / \mathrm{K}$.

After calculating the temperature coefficients for single subsamples, the calibration has been applied to the whole dataset. The transformation into a temperature independent frame is given by $U_{\mathrm{T}, i}\left(T=0{ }^{\circ} \mathrm{C}\right)=U_{\mathrm{T}, i}\left(T_{i}\right)-\kappa_{j} \cdot T_{i}\left[{ }^{\circ} \mathrm{C}\right]-\delta U_{\mathrm{T}, j}$. In the formula, $i$ are the different data points and $j$ the associated calibration. $\delta U_{\mathrm{T}}$ is an offset introduced to make the two SESAME calibrations comparable. The effect of the temperature calibration can be seen in Fig. 1.

The calibration has been checked by calculating the standard deviation of all sub-samples, which has been reduced significantly for most of the sub-samples. The standard deviation of the data points binned with respect to their temperature does not show a trend after calibration anymore and is reduced to the measurement precision for all temperatures. The initial threshold voltages measured by ESA-ESTEC [12] ( $-1.61 \mathrm{~V}$ for COSIMA and $-1.44 \mathrm{~V}$ for SESAME at $T=20^{\circ} \mathrm{C}$ ) are consistent with the values obtained from the datasets.

\subsection{Relation between dose and voltage measurements}

The threshold voltage can be connected to the accumulated dose $D$ in Silicon via $\Delta U_{\mathrm{T}}[\mathrm{V}]=\bar{\varepsilon} \cdot a_{1} \cdot D[\mathrm{~Gy}]^{a_{2}} . \Delta U_{\mathrm{T}}$ is the difference of the threshold voltage at a given time with respect to the non-irradiated device. The values $a_{1}=0.086 \pm 0.004$ and $a_{2}=0.78 \pm 0.03$ are obtained from ${ }^{60} \mathrm{Co}$ calibration measurements [8]. The dependency of the RadFET response on the radiation type and its energy is expressed in the mean efficiency $\bar{\varepsilon}$, which is given

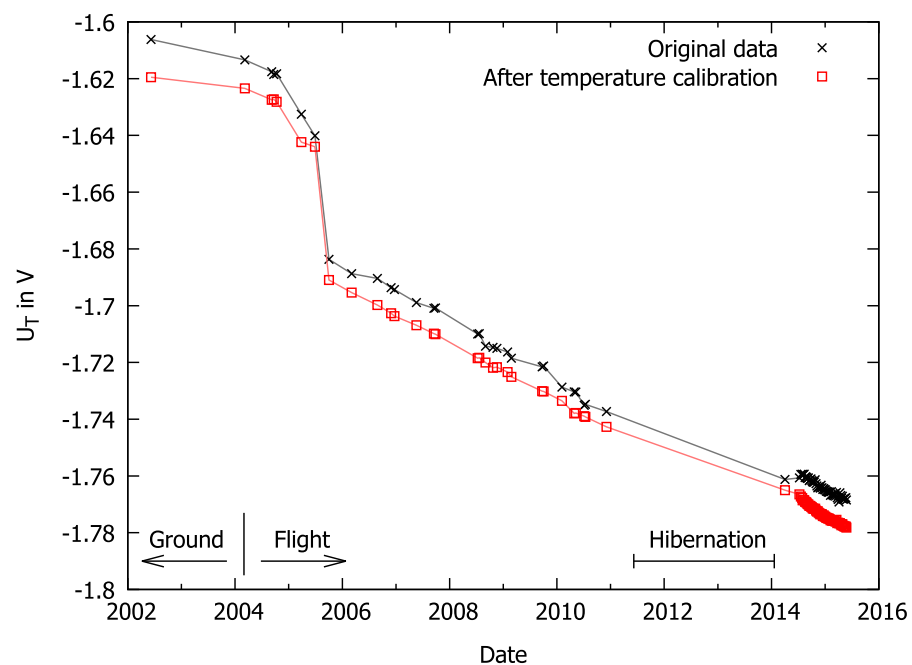

(a) by $\bar{\varepsilon}=\int_{0}^{\infty} \varepsilon(E) \cdot w(E) \mathrm{d} E$. The designation $\varepsilon(E)$ is the energy-dependent response for proton radiation and will be described in Section $4.5, w(E)$ is the normalized spectrum of the radiation environment at the position of the RadFETs and is used as a weighting function. It will be derived in Section 4.4. The exponent of the dose is expected to be independent of the radiation type and energy, since it describes an intrinsic property of the RadFET.

\subsection{Weighting function from radiation environment}

With the ESA SPENVIS interface [15], the radiation environment from solar and galactic protons has been calculated for the mission profile of Rosetta according to the ECSS space environment standard [1]. The standard recommends using the ISO 15390 model for GCR and the ESP solar proton model for SCR. The solar component has been calculated at a constant distance of $1 \mathrm{AU}$ and, deviating from the ECSS standard, scaled with a $r^{-2}$ behaviour according to the Rosetta orbit, for which we obtained a mean value of 0.369 for $r[\mathrm{AU}]^{-2}$. The ECSS standard recommends a scaling of $r^{-2}$ for $r<1 \mathrm{AU}$ and 1 for $r>1 \mathrm{AU}$ to obtain a conservative estimate for dose predictions. We expect the $r^{-2}$ scaling to model the dependency more accurately for an analysis of received dose levels. The impact of the different scaling on $\bar{\varepsilon}$ proves to be negligible. Other radiation components like the Earth flybys and ions with a higher charge than protons have been neglected due to their small contribution to the overall flux. The radiation spectrum inside the spacecraft has been calculated by using the CREME model suite [16] within SPENVIS and then normalized to an area of 1 to get the weighting function $w(E)$. The weighting functions are shown in Fig. 2. Both instruments are expected to have a comparable mean shielding $[8,17]$, although it varies for different solid angles. An equivalent shielding thickness of $4 \mathrm{~mm}$ Aluminium is suitable for both cases to calculate the mean efficiency. A more detailed discussion on the shielding thickness can be found in chapter 6.

The need for time-dependent weighting functions has been checked by calculating the expected fluences for different time steps after launch with SPENVIS and calculating the respective SCR suppression factors from the orbit. The different values of $\bar{\varepsilon}$ agree within $2 \%$, which is well below the efficiency model uncertainty and thus a constant weighting function is used. A single solar particle event can produce almost the total dose of a mission and

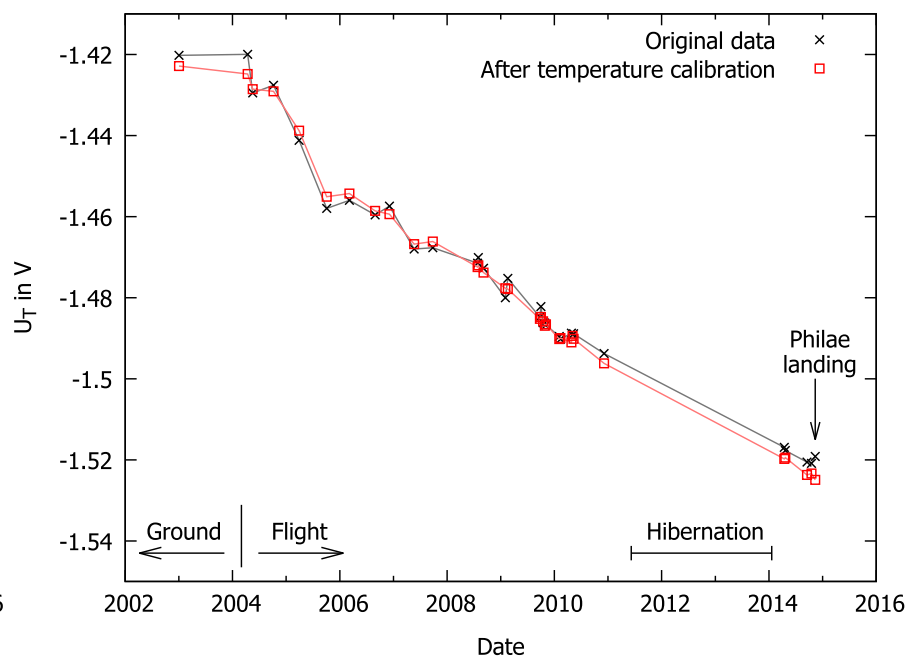

(b)

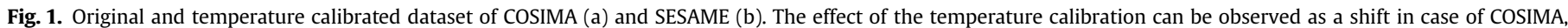
since the instrument was typically warmer than the reference temperature of $T=0{ }^{\circ} \mathrm{C}$. A reduction of the spread is visible for both instruments. 


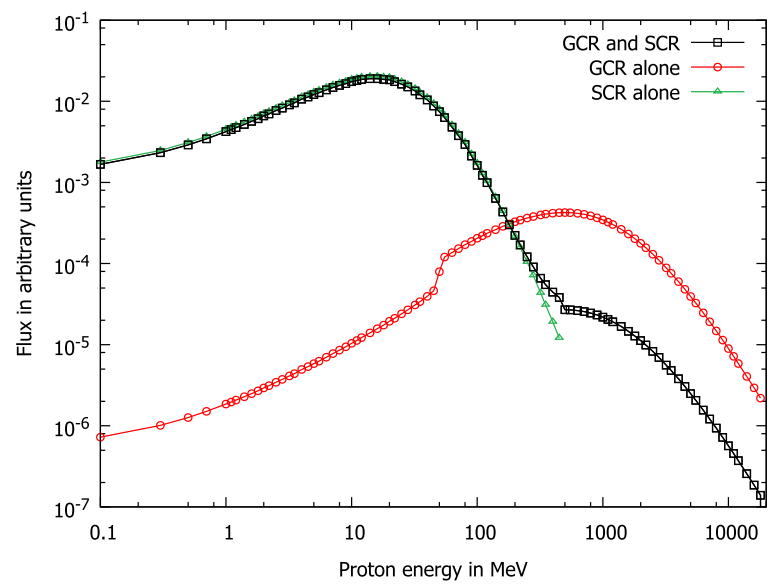

Fig. 2. Normalized spectra of GCR, SCR and the sum of both components according to their relative fluences for the mission epoch behind $4 \mathrm{~mm} \mathrm{Al} \mathrm{shielding.} \mathrm{The}$ spectra are used as weighting functions and have been calculated with the ESA SPENVIS interface. The main contribution to the flux is given by the solar component, which has a softer energy spectrum than the GCR component.

therefore various known energy spectra of SPEs have been analysed. The difference in $\bar{\varepsilon}$ due to strong SPEs can be neglected. The effect of an exceptionally quiet Sun with the SCR fluence reduced to $10 \%$ of its expected contribution to the total fluence has been checked as well and yields maximally a 6\% higher value of $\bar{\varepsilon}$.

\subsection{Mean RadFET efficiency}

Different experiments with proton beams at various energies have been performed with RadFETs previously in order to measure the energy dependency of the response $[10,18]$. The high energies of the GCR component have not been reached. A spread of 3-4\% in the RadFET responses also from RadFETs of the same manufacturer and out of the same production cycle is present [10]. We have derived an efficiency model from these measurements and theoretical considerations, which expect no further increase in response above $\approx 60 \mathrm{MeV}$ [19]. The model is given by

$\varepsilon(E)=\left\{\begin{array}{ll}49 \%+0.65 \% \cdot E[\mathrm{MeV}] & \text { if } E<60 \mathrm{MeV} \\ 88 \% & \text { else }\end{array}\right.$.

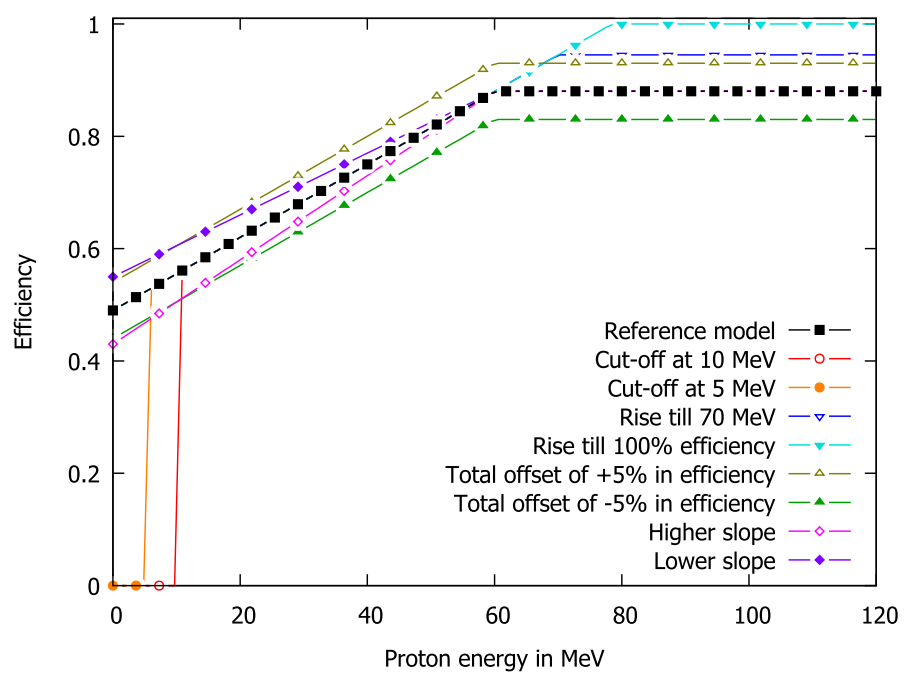

(a)
The reference model is consistent with an efficiency of $53 \%$ to $65 \%$ for $17.4 \mathrm{MeV}$ protons determined in [8], where a RadFET out of the same manufacturing cycle has been tested. The uncertainties are taken into account by eight additional models to determine the size of the error bars, especially covering different hypotheses for the extrapolation to the highest and lowest energies, were no measurements are available. For example, models with a low energy cut-off have been applied to account for the limited range of protons in the device. An overview of the models is shown in Fig. 3a.

The mean efficiency obtained by the efficiency model and the radiation environment for a shielding thickness of $4 \mathrm{~mm}$ Aluminium is given by $\bar{\varepsilon}=0.72 \pm 0.10$ (see Fig. 3b). The error on $\bar{\varepsilon}$ includes the predictions of the efficiency reference model for all shielding thicknesses up to $d=20 \mathrm{~mm}$.

\section{Results}

The time evolution of the dose is given in Fig. 4. It follows a linear increase with additional steps due to strong solar particle events (the first in January 2005 at a distance of $1.1 \mathrm{AU}$ from Rosetta to the Sun and the second in September 2005 at a distance of 1.6 AU). The total dose on 2014-11-14 (3909 days after launch) is 3.2 $\pm 0.6 \mathrm{~Gy}$ in case of COSIMA and $1.9 \pm 0.4 \mathrm{~Gy}$ in case of SESAME. For the last data subsample of COSIMA on 2015-05-26 (4102 days after launch), a dose of $3.3 \pm 0.7 \mathrm{~Gy}$ was obtained. Linear regressions of the dose evolution after the second SPE yield a slope of $0.2196 \pm 0.0008 \mathrm{~Gy} /$ year in case of COSIMA and $0.179 \pm 0.003 \mathrm{~Gy} /$ year for SESAME. No significant dose increase due to the Earth flybys has been observed, which is in accordance with the expectation due to the low energy of the electrons and ions in the radiation belts of the Earth as well as the short time periods of the flybys.

The dose changes due to the SPEs are given in Table 1. The ratio of the increases between COSIMA and SESAME is $2.5 \pm 1.3$ for the first SPE and $5.3 \pm 1.0$ for the second SPE. In both cases COSIMA had the higher increase.

No evidence for long-term fading has been found in the dataset. There might be short-term fading, which could be invisible due to the typical period of six months between the different data points. The drift of the voltage signal in the first minute after

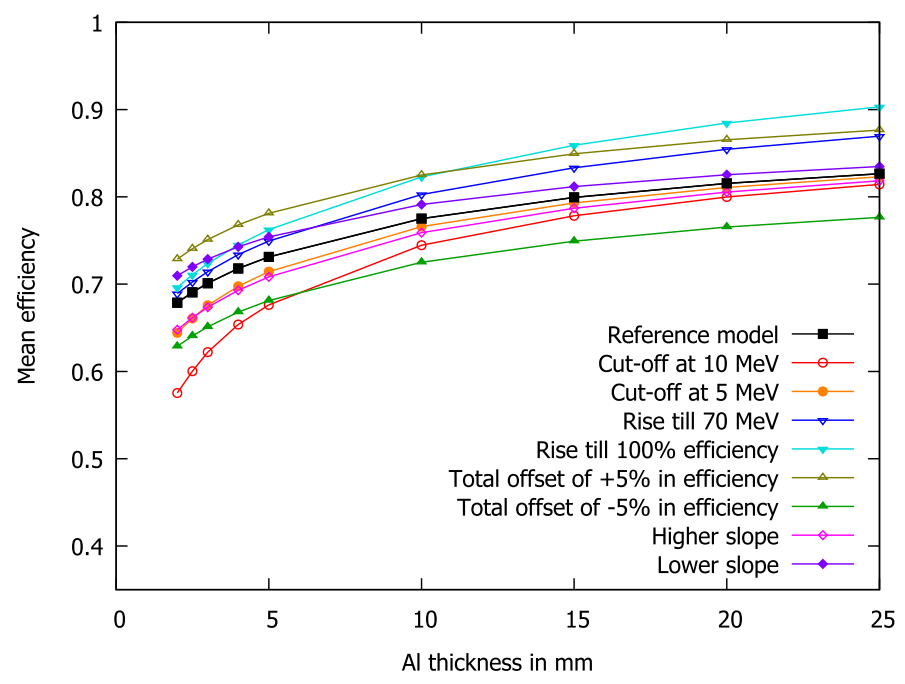

(b)

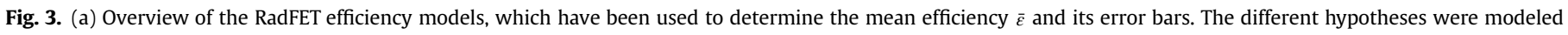

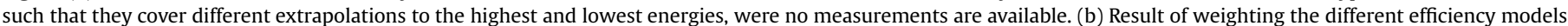
with the expected proton spectrum (compare to Fig. 2) to obtain the mean efficiency. 


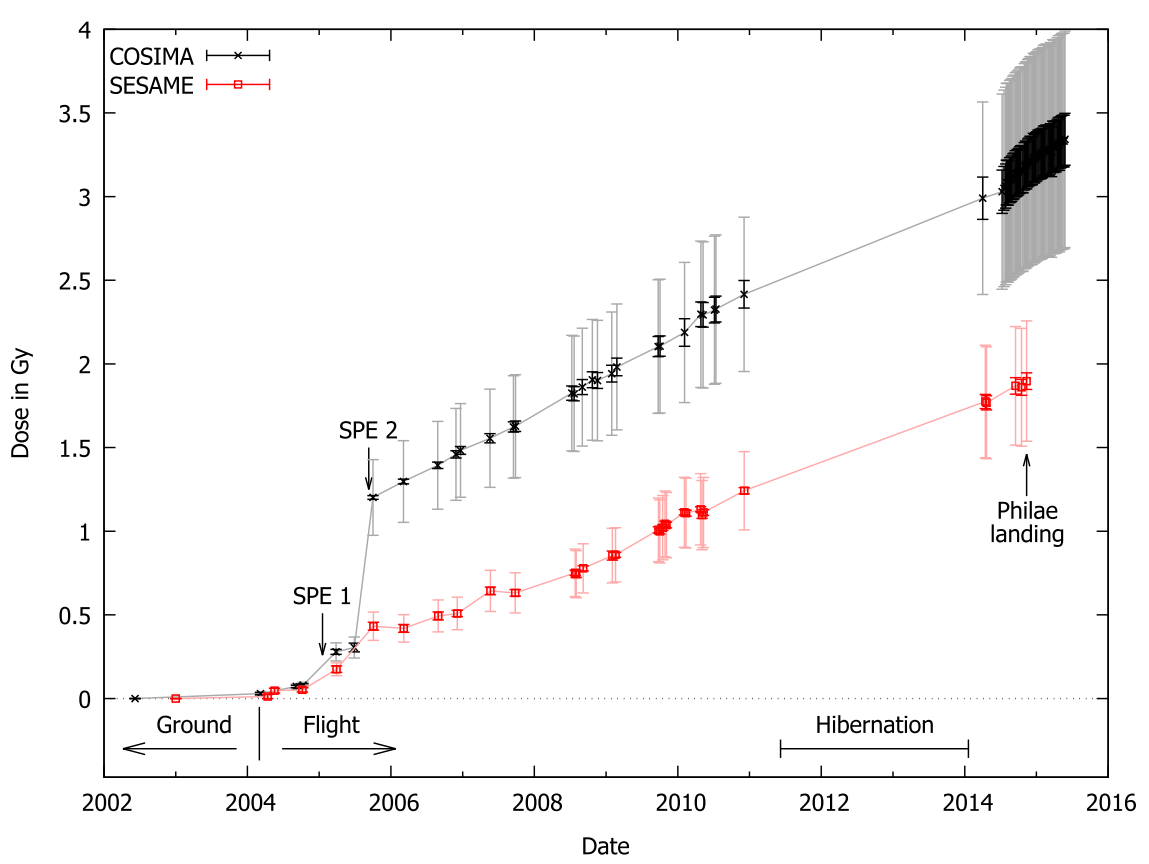

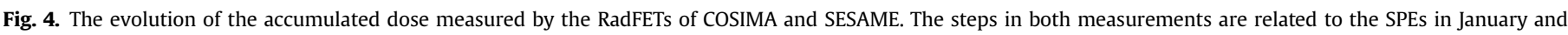

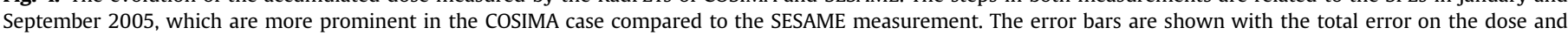
additionally without the scaling uncertainties placed by $\bar{\varepsilon}$ and $a_{1}$.

Table 1

Dose changes due to the two strong solar particle events in 2005. The second SPE has a major impact on the COSIMA measurement. The error bars are given without the scaling uncertainty.

\begin{tabular}{lll}
\hline Instrument & $\Delta D$ due to first SPE & $\Delta D$ due to second SPE \\
\hline COSIMA & $0.10 \pm 0.02 \mathrm{~Gy}$ & $0.84 \pm 0.03 \mathrm{~Gy}$ \\
SESAME & $0.04 \pm 0.02 \mathrm{~Gy}$ & $0.16 \pm 0.03 \mathrm{~Gy}$ \\
\hline
\end{tabular}

turning on the supply voltages might have an effect in the order of one percent on the accuracy of the measurements and cannot be excluded for all readout variants except one for SESAME, were the signal has been measured at least one minute after turning on the supply voltages.

\section{Pre-launch dose predictions}

The predicted radiation environment of the Rosetta mission has been calculated in detail for the mission target 46P/Wirtanen in [20], including galactic cosmic rays, solar protons and contributions from the radiation belts of the Earth. The fluences of interplanetary solar protons were considered with statistical models developed at the Jet Propulsion Laboratory (JPL). JPL models distinguish between active and inactive phases of a solar cycle.

The JPL-85 solar proton model has been used in favour to the newer JPL-91 model, since it predicts higher fluences of low energy protons and thus greater damage to low shielded components like solar cells. Two active solar phases were identified for the Rosetta mission to 46P/Wirtanen, for which the orbit has been approximated as being circular at $1.2 \mathrm{AU}$ (first 3.5 years of the mission) and 2.3 AU (last two years). The solar proton fluences have been calculated at a $90 \%$ confidence level for not being exceeded and scaled according to a $r^{-2}$ dependency.

The obtained particle fluence spectra were used to calculate the expected maximum dose in a Silicon target as a function of spherical Aluminium shell thickness. For a typical shielding
Table 2

Predicted maximum dose (90\% confidence level) in an Aluminium box with different wall thicknesses at the COSIMA position [17]. The shielding of the spacecraft alone corresponds typically to a shielding of $2 \mathrm{~mm}$ Aluminium.

\begin{tabular}{ll}
\hline Wall thickness in mm & Dose in Gy \\
\hline 0.5 & 80 \\
1.0 & 58 \\
1.5 & 45 \\
2.0 & 36 \\
\hline
\end{tabular}

thickness of $4 \mathrm{~mm}$, a dose of $50 \mathrm{~Gy}$ was obtained. Corresponding values based on other predictions of the radiation environment are 29 Gy (using JPL-91 solar proton fluence at 90\% confidence level [8]) and $90 \mathrm{~Gy}$ (applying 90\% confidence level for the first and 95\% for the final active phase [21]). The considerable range of values shows how much the calculated dose levels that will likely not be exceeded depend on the applied engineering model and the required confidence level.

The dose profile depending on the thickness of a spherical Aluminium shell has been used in [17] for a raytracing analysis of Rosetta's geometry. It yielded the expected dose at various positions in the spacecraft, considering different thicknesses of further shielding (for example due to electronics boxes). The JPL- 85 model has been applied for the solar protons and an isotropic proton flux was assumed according to the ECSS standard [1].

A dose of $58 \mathrm{~Gy}$ was obtained inside a box made of $1 \mathrm{~mm}$ Aluminium walls at the position of COSIMA central electronics. The box should be a suitable representation of the more complex housing of the COSIMA instrument (a comparison with different wall thicknesses is shown in Table 2).

For a central position in the lander within $2 \mathrm{~mm}$ additional shielding, a dose of 62 Gy was calculated in [17]. The full geometry of the lander with empty electronics boxes was simulated in [8] and for SESAME central electronics $56 \mathrm{~Gy}$ were obtained using the JPL-85 model at $90 \%$ confidence level (and 33 Gy using the JPL-91 model). Considering the results obtained with the same particle fluence spectra only, very similar dose levels were thus calculated 


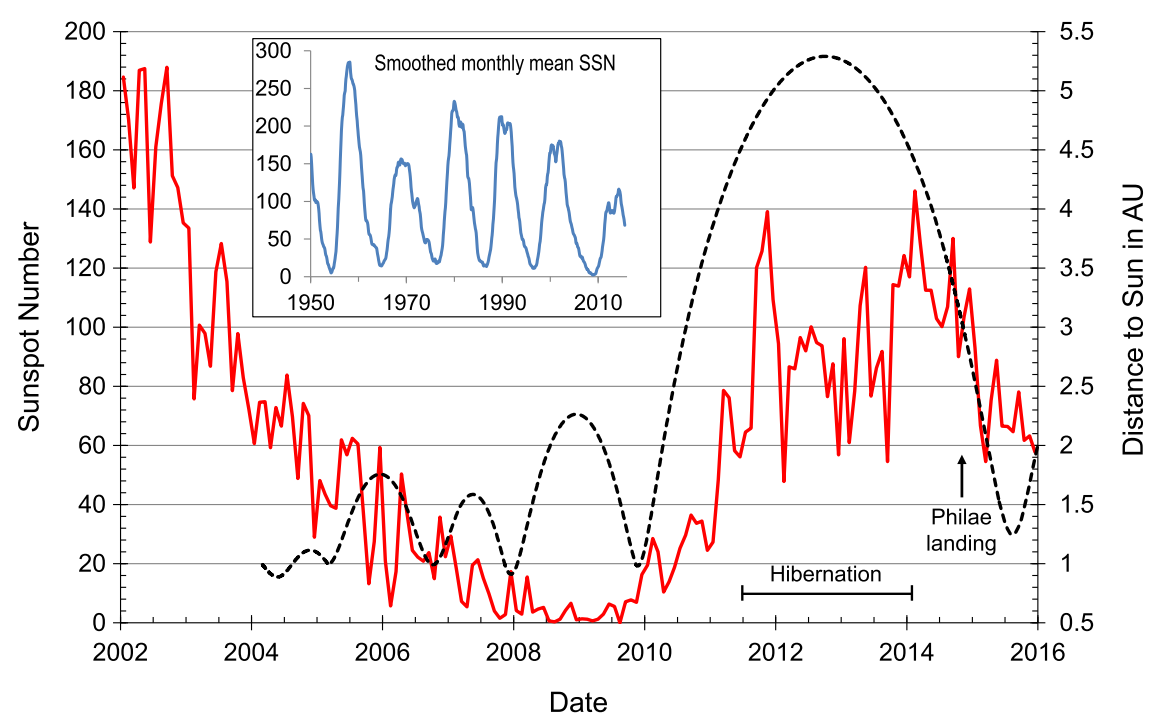

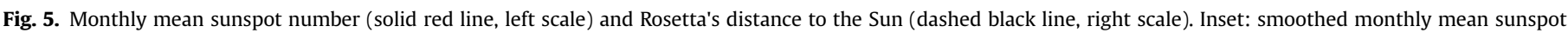

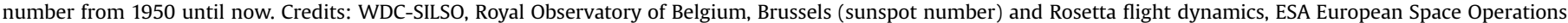
Centre, Darmstadt (orbit). (For interpretation of the references to colour in this figure caption, the reader is referred to the web version of this paper.)

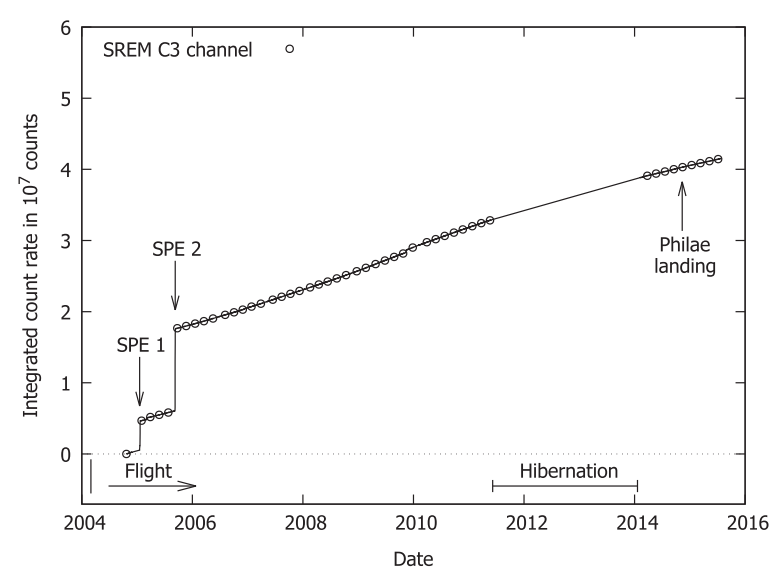

Fig. 6. Integrated count rate of the SREM coincidence channel $\mathrm{C} 3$, which is sensitive to protons of energies between $76 \mathrm{MeV}$ and $450 \mathrm{MeV}$ [33]. The data points in the figure indicate the coverage periods of the SREM dataset, measurements were taken with a much higher frequency. The count rates were obtained from [28] and are available since 2004-10-21. Our integration was performed by summing over all measurements multiplied by the time till the subsequent measurement. Count rates of periods in which the SREM was turned off (especially the hibernation) are approximated by the mean value of 1000 data points before and after the interruption. The C3 channel is an example for several SREM channels (especially the ones sensitive to protons) which show a linear increase of the integrated count rate, which can be associated to GCR, as well as the two observed SPEs. The slight changes in the slope of the GCR component are expected to be caused by the anticorrelation with the solar activity, which became higher during Deep Space Hibernation (see Fig. 5).

for COSIMA and SESAME central electronics.

A full revision of the predicted maximum doses for the instruments to the final mission target 67P/CG has not been found. The expected proton fluences calculated with the JPL-91 model at 90\% confidence level [21] and the resulting dose profiles depending on shielding thickness [22] are available. For a Silicon target surrounded by a $4 \mathrm{~mm}$ thick spherical Aluminium shell a dose of $67 \mathrm{~Gy}$ was calculated, which is well in the range of corresponding values calculated for the mission to $46 \mathrm{P} /$ Wirtanen. The updated mission radiation environment might cause a minor change of relative dose levels for SESAME and COSIMA, but a full shielding analysis, based on an isotropic particle flux, would probably still yield similar dose levels for the two instruments.
Predictions of solar activity commonly refer to sunspot statistics. Although reliable observational sunspot data covers almost three centuries, the forecast of amplitude and duration of a future cycle is still difficult. In June 2003, when the radiation analysis for the mission to 67P/CG was performed, enhanced solar activity due to the beginning of solar cycle 24 was expected for end of 2006 latest [21]. Actually there was minimal activity until early 2010 and cycle 24 became the weakest solar cycle since 1906 [23]. The high GCR fluxes due to the deep solar minimum (see chapter 7) were reduced during the maximum period of cycle 24, when Rosetta's distance to the Sun was large. Solar activity (in terms of the sunspot numbers) was low when Rosetta was closer to the Sun, which reduced the risk of severe radiation exposure due to SPE (Fig. 5).

\section{Discussion}

The accumulated dose of the RadFETs is one order of magnitude lower than the predictions for 67P/CG at a confidence level of $90 \%$ for not being exceeded. A preliminary analysis showed that the Rosetta SREM observed a fluence which is a factor of 5 to 10 (depending on the energy) below the JPL-91 predictions, too [24]. The Sun has been exceptionally quiet during the journey of Rosetta $[25,26]$ (compare to Fig. 5), giving an explanation of the low dose. The launch to the previous mission target, 46P/Wirtanen, was planned for January 2003. In late 2003 a strong solar particle event occurred [27], which was one of the largest SPEs observed in the last 50 years. The SPE proton fluence has been measured by the SREM on-board the spacecraft Integral [28], which is situated in an Earth orbit. Rosetta would have been at approximately $1 \mathrm{AU}$ to the sun during that time, making the Integral and Rosetta environment comparable. The SPE would have led to an increase of the accumulated dose by a factor of $2-3$ compared to the 67P/CG measurement. This shows that single SPEs can make up almost the total radiation environment of a space mission.

The predictions at $90 \%$ confidence level appear to be conservative, in spite of the Sun being exceptionally quiet. The caution in the predictions could be driven by the importance of Rosetta as a cornerstone mission of ESA and the 11-year duration of the flight, in which Rosetta may have also encountered a more severe radiation environment. Rosetta relies fully on the power 
generation of solar panels, which are vulnerable and particularly exposed to cosmic radiation. Considering the 90\% confidence level predictions as a radiation dose budget, the Rosetta spacecraft is well inside the margins and should not exceed them till the planned mission end.

Both RadFETs measured a linear increase of the dose, which we mainly attribute to GCR protons and which is present similarly for the integrated count rates of the Rosetta SREM (see Fig. 6). The difference in the slopes for COSIMA and SESAME is about 23\%. It might be an effect of the different response from RadFET manufacturing or slightly different GCR radiation environments at the position of the electronics due to different shielding. We have not found information on the response spread of RadFETs at energies above $60 \mathrm{MeV}$. Unresolved minor SPE could have an effect on the slopes for the two instruments as well, since COSIMA detected the two SPEs with a higher dose increase than SESAME. SREM data shows only small contributions of minor SPEs to the count rates, though.

The linear regressions refer to dose values collected during a period of more than eight years after the second SPE in September 2005. The low frequency of RadFET readouts and the measurement precision do not support the evaluation of dose rate changes during this period, which included the highest GCR fluxes of the space age due to the deep and extended solar minimum in 20082010 [26]. The CRaTER microdosimeter of the Lunar Reconnaissance Orbiter (LRO) observed a decrease of the GCR dose rate by a factor of 2 from the mission start in June 2009 to the end of 2014 [29]. Spacecraft and ground based data [30] suggest that GCR intensity was about to come back to the level of late 2005, when the Mars Science Laboratory (MSL) spacecraft, containing the Curiosity rover, was on its way to Mars.

Measurements with Curiosity's Mars Radiation Assessment Detector (RAD) were taken from 2011-12-06 to 2012-07-14 and yielded a GCR dose rate of $0.121 \pm 0.008 \mathrm{~Gy} /$ year on average (corresponds to $332 \pm 23 \mu \mathrm{Gy} /$ day [31]). This is considerably lower than the mean dose rates inferred from the RadFET measurements, which can mainly be attributed to the different measuring periods. Additionally, the radial GCR gradient of about 4.5\%/AU [32] contributes to the higher RadFET dose rates. The mean shielding thickness around RAD [31] was larger than the mean shielding of the RadFETs; the impact of the shielding on dose rates depends on material composition and might, in spite of the production of secondary particles in the shielding by the high energy GCR ions, still contribute to the lower dose rate at Curiosity's RAD. The GCR dose rate determined with RAD might thus be considered as a tentative lower reference value for the RadFET dose rates. The transit of MSL to Mars took place during the active period of solar cycle 24 , but GCR predominantly contributed to the accumulated dose [31]. The most intense SPE observed by LRO/CRaTER contributed only about $14 \%$ to the total dose accumulated during 5.5 years, which include a large fraction of the recent solar maximum [29]. The relatively low dose contribution from solar protons measured with the SESAME and COSIMA RadFETs was thus observed by other missions as well. The two spacecraft did, however, not encounter the major SPE in 2005 seen by Rosetta.

The detection of the two SPEs yielded much higher differences in the dose changes $\Delta D$ between the RadFETs compared to the ratio between the linear slopes. It is not expected to be explained by the different RadFET response alone. The ratios between the dose increases of COSIMA and SESAME $\Delta D$ (COSIMA) $/ \Delta D$ (SESAME) for the two SPEs are incompatible with each other as well. Measurements with other instruments show that the second SPE had a softer energy spectrum than the first and, due to a higher time resolution, that the two SPEs seen by the RadFETs consisted itself out of several events [34,35]. In case of the first SPE, the sub-event of 2005-01-20 had an exceptionally hard spectrum. The different spectra of the SPEs in addition to the slightly different shielding of the instruments give a possible explanation of the observed difference between COSIMA and SESAME, although it still seems to be large.

Another explanation of the SPE dose changes for the two instruments to be considered is the possible directionality of the SPEs. COSIMA is placed on the $+Z$ plane of Rosetta at the edge to the $+X$ plane, which has been oriented towards the Sun during flight [36]. Philae (and with it SESAME) is placed on the $-X$ plane [36] and therefore on the opposite side of the Sun. SESAME is shielded against the direction of the Sun by the Rosetta spacecraft, whereas COSIMA does not have a high shielding in this direction compared to the other directions [17]. Solar particle events with a transient high directionality have been observed before by other space missions [37-41]. It is possible, that the second SPE hit the spacecraft in a way, that SESAME has been shielded and COSIMA not (that is from the half-sphere in which the Sun is centred). The first SPE might have been more isotropic or hit the spacecraft from a direction in which both instruments are more equally sensitive. A conclusive statement on the origin of the observed differences in the SPE doses is not possible based on the RadFET measurements alone. The understanding might be enhanced by a more detailed analysis of the SREM data due to the various energy channels, the opening angle of the aperture and the position of the instrument. It would therefore be desirable to perform a joint analysis in the future, since it is out of the scope of this article.

The solar particle event in September 2005 hit Rosetta at the beginning of a weekly non-coverage period. During the next signal acquisition, the active star tracker of Rosetta was found to be crashed in INIT mode. The attitude of the spacecraft was obtained for six days using gyroscopes only, accumulating a drift of about $0.7^{\circ}$. Fortunately, the misalignment of the high gain antenna was still small enough to allow the RF signal to be received on Earth [42]. These problems emphasize the fact that the radiation protection of a spacecraft remains an important issue to stay fully functional during the whole mission.

\section{Acknowledgements}

The RadFETs mounted in the central electronics of COSIMA and SESAME were provided by Ali Mohammadzadeh (ESA-ESTEC). We thank as well Petteri Nieminen (ESA-ESTEC) for useful comments to an earlier internal report on COSIMA and SESAME RadFET measurements. Hugh Evans (ESA-ESTEC) sent us preliminary information on proton flux measurements with Rosetta/SREM. Additionally we thank two anonymous reviewers for their comments and suggestions.

K. Thiel and H.-H. Fischer gratefully acknowledge the support of RadFET irradiation experiments by the deans and the staff of the following institutions: Institute of Nuclear Chemistry at the Jülich Research Centre (Forschungszentrum Jülich), in particular the staff of the cyclotron unit, the Institute of Physical Chemistry, University of Cologne, enabling ${ }^{60} \mathrm{Co}$ irradiations. Special thanks to Uwe Otto, Nuclear Chemistry Department Electronics Workshop at the University of Cologne.

\section{References}

[1] ESA, ECSS-E-ST-10-04C - Space engineering - Space environment, 2008.

[2] B. Heber, Modulation of galactic and anomalous cosmic rays in the inner heliosphere, Adv. Space Res. 27 (2001) 451-460.

[3] J.A. Simpson, Elemental and isotopic composition of the galactic cosmic rays, Annu. Rev. Nucl. Part. Sci. 33 (1983) 323-382.

[4] K.J. Seidensticker, et al., SESAME - an experiment of the Rosetta lander Philae: objectives and general design, Space Sci. Rev. 128 (2007) 301-337. 
[5] J. Kissel, et al., COSIMA high resolution time-of-flight secondary ion mass spectrometer for the analysis of cometary dust particles onboard Rosetta, Space Sci. Rev. 128 (2007) 823-867.

[6] H. Evans, et al., Results from the ESA SREM monitors and comparison with existing radiation belt models, Adv. Space Res. 42 (2008) 1527-1537.

[7] W. Hajdas, et al., Standard radiation environment monitor - data repository and web based data analysis tools, in: 33rd International Cosmic Ray Conference, 2013, 〈http://www.cbpf.br/ icrc2013/papers/icrc2013-1224.pdf).

[8] H.-H. Fischer, Software-Entwicklung für das SESAME-Experiment der RosettaKometenmission und Untersuchungen zum RADFET-Dosimeter, Dissertation, University of Cologne, 2002.

[9] A. Haran, et al., Temperature effects and long term fading of implanted and unimplanted gate oxide RADFETs, IEEE Trans. Nucl. Sci. 51 (2004) 2917-2921.

[10] A. Jaksic, Report on activities related to MTSL RADFET dosimeters development and supply during the 2002/2003 programme, internal ESA Document 2004

[11] Tyndall National Institute, 4kÅ Implanted Gate Oxide SOLID STATE DOSIMETER, website, 2015, 〈http://project.tyndall.ie/radfets/ds400i.html〉.

[12] H. Henkel, RadFET Bestands-Update, Internal Technical Note, 2001.

[13] A. Accomazzo, Rosetta Deep Space Hibernation PL Issues, RO-ESC-TN-5048, Internal Rosetta Project Document, 2010.

[14] R. Schulz, et al. (Ed.), ROSETTA - ESA's Mission to the Origin of the Solar System, Springer, New York, 2009

[15] ESA, SPENVIS - Space Environment, Effects, and Education System, Website, 2013, 〈https://www.spenvis.oma.be/〉.

[16] ESA, CREME Software Description, Website, 2013, 〈https://spenvis.oma.be/ help/background/creme/creme.html>.

[17] Astrium, Radiation Analysis, RO-DSS-AN-1004, internal Rosetta Project Document, 2002.

[18] R.L. Pease, M. Simons, P. Marshall, Comparison of pMOSFET total dose response for Co-60 gammas and high-energy protons, IEEE Trans. Nucl. Sci. 48 (2001) 908-912.

[19] T.R. Oldham, Analysis of damage in MOS devices for several radiation environments, IEEE Trans, Nucl. Sci. 31 (1984) 1236-1241.

[20] H. Evans, Rosetta Radiation Environment - 2, esa/estec/wma/he/Rosetta-97/2, internal ESA Document, 1997.

[21] H. Evans, Rosetta Radiation Environment: Churyumov-Gerasimenko, esa/estec/tos-em/03-029/he, internal ESA Document, 2004.

[22] W. Keil, Radiation Impact Comet Churyumov-Gerasimenko vs. Wirtanen, RODSS-TN-1199, internal Rosetta Project Document, 2003.

[23] Royal Observatory of Belgium, Yearly mean and monthly smoothed sunspot number - SILSO, Website, 2016, 〈http://www.sidc.be/silso/yearlyssnplot〉.

[24] H. Evans, personal communication, Accumulated Dose of the Rosetta SREM (2015).

[25] C.T. Russell, J.G. Luhmann, L.K. Jian, How unprecedented a solar minimum? Rev. Geophys. 48 (2010) RG2004.
[26] R.A. Mewaldt, et al., Record-setting cosmic-ray intensities in 2009 and 2010, Astrophys. J. Lett. 723 (2010), L1.

[27] R.A. Mewaldt, et al., Proton, helium, and electron spectra during the large solar particle events of October-November 2003, J. Geophys. Res. 110 (2005), A09S18.

[28] ESA-ESTEC, SREM Data Access, Website, 2015, 〈http://spitfire.estec.esa.int/se dat/dplot_odi.html>.

[29] J.E. Mazur, et al., Update on radiation dose from galactic and solar protons at the moon using the LRO/CRaTER microdosimeter, Space Weather 13 (2015) 363-364.

[30] D. Matthiä, M.M. Meier, G. Reitz, Numerical calculation of the radiation exposure from galactic cosmic rays at aviation altitudes with the PANDOCA core model, Space Weather 12 (2014) 161-171. The reference includes spacecraft and ground based data as well as derived values of a solar modulation parameter. Neutron monitor data are also available at $\langle$ http://www.nmdb.eu/ $\rangle$. Data of the spacecraft Advanced Composition Explorer (ACE) at 〈http://www. srl.caltech.edu/ACE/ $\rangle$.

[31] C. Zeitlin, et al., Measurements of energetic particle radiation in transit to Mars on the Mars Science Laboratory, Science 340 (2013) 1080-1084.

[32] J. Gieseler, et al., The radial gradient of galactic cosmic rays: Ulysses KET and ACE CRIS measurements, in: 30th International Cosmic Ray Conference, vol. 1, 2008, pp. 571-574.

[33] I. Sandberg, et al., Unfolding and validation of SREM fluxes, IEEE Trans. Nucl. Sci. 59 (2012) 1105-1112.

[34] H. Evans, Solar Cell Radiation Equivalent Fluences for Rosetta Mission, TECEES/2006.28/HE, internal Rosetta Project Document, 2006.

[35] R.A. Mewaldt, et al., Solar-particle energy spectra during the large events of October-November 2003 and January 2005, in: 29th International Cosmic Ray Conference, vol. 1, 2005, p. 111.

[36] Astrium, Rosetta Users Manual (Vol. 1), 2003. RO-DSS-MA-1001, internal Rosetta Project Document.

[37] J. Torsti, E. Riihonen, L. Kocharov, The 1998 May 2-3 Magnetic Cloud: an interplanetary "highway" for solar energetic particles observed with $\mathrm{SOHO}$ ERNE, Astrophys. J. Lett. 600 (2004), L83.

[38] R.A. Leske, et al., Large proton anisotropies in the 18 August 2010 solar particle event, Sol. Phys. 281 (2012) 301-318.

[39] J. Torsti, et al., Recurrence of energetic particle flux anisotropy as observed by ERNE on 9 July 1996, Sol. Phys. 175 (1997) 771-784.

[40] T. Sahla, J. Torsti, SOHO/ERNE measurement of directional proton intensities during the coronal mass ejections in April and May 1997, in: 26th International Cosmic Ray Conference, vol. 6, 1999, p. 332.

[41] J. Torsti, T. Sahla, Development of directional flux anisotropy during the solar event on June 16, 1998, in: 26th International Cosmic Ray Conference, vol. 6, 1999, p. 336.

[42] ESA, Status Report No. 48 - Solar Flare Interacts with Rosetta, Website, 2005 〈http://sci.esa.int/rosetta/37921-rosetta-status-report-no-48/〉. 